\title{
Temporal uncertainty and the "refractoriness" of the human vertex evoked potential
}

\author{
D. G. WASTELL \\ Department of Medical Computing, Manchester University Medical School \\ Manchester M13 9PL, England \\ D. KLEINMAN \\ Department of Psychology, University of Durham, Durham DH1 3LE, England \\ and \\ A. MACLEAN \\ MRC Applied Psychology Unit, Cambridge CB2 2EF, England
}

\begin{abstract}
The so-called refractoriness of the N1-P2 wave of the vertex evoked potential is examined using an experimental procedure based on an analog clock that enables control over temporal uncertainty. It is shown that in both the visual and auditory modalities, variations in temporal uncertainty underlie refractoriness, although in the auditory modality, another variable, designated "firstness," may also play a role. The potential value of the clock paradigm is also explored in time-locking emitted potentials in the missing-stimulus paradigm.
\end{abstract}

For a period of several seconds following its evocation, the N1-P2 complex of the human vertex evoked brain potential (auditory and visual) exhibits a state of refractoriness. Depending on the paradigm employed and the presuppositions of the investigator, this phenomenon of refractoriness is known as either fast (shortterm) habituation (Fruhstorfer, Soveri, \& Jarvilehto, 1970 ) or temporal recovery (Davis, Mast, Yoshie, \& Zerlin, 1966). Concerning the mechanism of the phenomenon, Bess and Ruhm (1972) and Wastell and Kleinman (1980) (using dichotic and dichoptic presentation, respectively) have shown a central rather than a peripheral level to be involved for both the auditory and visual modalities. In this paper, we report a program of short experiments designed to shed further light on the underlying basis of evoked-potential (EP) refractoriness.

\section{EXPERIMENT 1}

If the "refractoriness" of the vertex EP is indeed a reflection of a state of refractoriness in the neural networks activated by the stimulus, then it is reasonable to expect the degree of decrement induced to be a positive function of the numbers of neural elements held in common by the stimuli and, hence, to reflect their physical similarity. Experiment 1 examined this prediction.

Details of EEG recordings are as follows: EEG was recorded monopolarly from the vertex (referred to earlobe or mastoid) using $\mathrm{Ag}-\mathrm{AgCl}$ electrodes. Amplifier time constant was .1 sec. Poststimulus epochs, $500 \mathrm{msec}$ in duration, were averaged, and peak-to-peak N1-P2 amplitude was measured. (These recordings details are common to all the experiments reported in this paper.)

Four subjects were run under two conditions. A tachistoscopically presented checkerboard (radius = $.75 \mathrm{~cm}$, duration $=40 \mathrm{msec}$ ) was preceded either by an identical checkerboard or by a solid concentric annulus of such inner radius $(1.75 \mathrm{~cm})$ that it did not overlap the checkerboard. Twenty-five such pairings of stimuli were presented in a block for each condition. Conditions were counterbalanced across subjects. Fixation was to a central spot. Subjects were instructed to attend passively to the stimuli. Stimuli in a pair were separated by an interstimulus interval (ISI) of $1 \mathrm{sec}$, and pairs were separated by an interval of $10 \mathrm{sec}$ to allow complete recovery of N1-P2 amplitude. Irrespective of the physical similarity of the prior stimulus, N1-P2 was found to be equally depressed in both conditions $(F<1)$.

\section{EXPERIMENT 2}

The results of Experiment 1 suggest that stimulus dimensions other than physical ones underlie EP refractoriness. One dimension that rapid stimulus repetition will certainly affect is temporal uncertainty. Given the apparent sensitivity of EP amplitude to temporal uncertainty (McCarthy \& Donchin, 1976), a plausible hypothesis is that EP refractoriness (which denotes an amplitude depression due to recent prior stimulation) is due to reduced temporal uncertainty when intervals between stimuli are short. With temporal uncertainty brought under control, "refractoriness" should then be abolished. This was the prediction tested in Experiment 2. 
Time into

trial (secs)

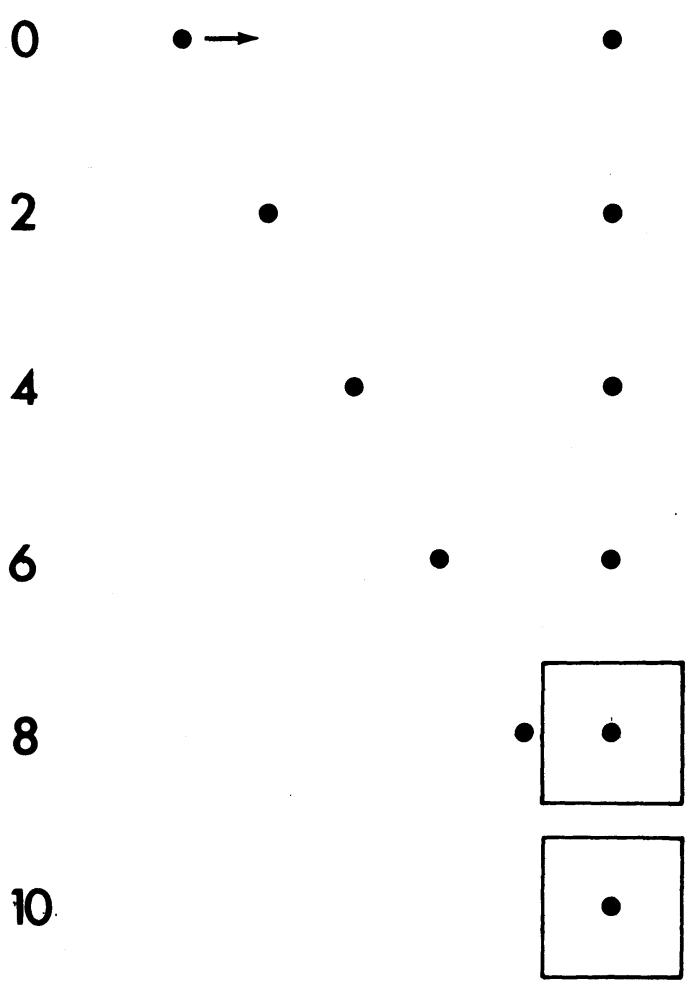

Figure 1. The clock paradigm is illustrated by a typical series of events. The left-hand spot moves uniformly toward the right-hand stationary fixation spot. On this trial, the checkerboard (represented by the square) occurs twice: at the end of the spot's traverse (RT required) and $2 \mathrm{sec}$ before this point (the irrelevant prior stimulus). Subjects viewed the display from a distance of $5 \mathrm{ft}$, at which its total area subtended $5 \mathrm{deg}$ at the eye.

Control over temporal uncertainty was achieved by using an analog "clock" to time the occurrence of events (see Figure 1). An oscilloscope spot was driven across a screen at a slow uniform rate, reaching the end of its traverse at the point of fixation in $10 \mathrm{sec}$. At this point, the stimulus (a checkerboard pattern) was presented (with $50 \%$ probability), to which a reaction time (RT) was required. At a random interval $(1$ to $3 \mathrm{sec}$ ) before this time, the checkerboard also could occur on a random $50 \%$ of trials. No RT to this event was required. Thus we have a situation in which a stimulus (the imperative one) may occur either a short interval (on average, $2 \mathrm{sec}$ ) after its prior presentation or after a long "full-recovery" interval (when the random event does not occur), but, via the clock, with equal temporal uncertainty in both cases. Our question, then, was whether, in the former case, in which refractoriness would usually be expected, it would be abolished by controlling temporal uncertainty. Six subjects were run. No such refractoriness was found, with N1-P2 amplitude being unaffected by the interpolated stimulus $[\mathrm{t}(5)=.39, \mathrm{p}>.5]$. We conclude the refractoriness of the visual EP to reflect reduced temporal uncertainty at short ISIs.

\section{EXPERIMENTS 3 AND 4}

Experiment 3 comprised an attempt to generalize the result of Experiment 2 to the auditory modality, in which the majority share of temporal recovery and habituation research has been conducted. The "clock" paradigm was again applied. Hoping also to abolish refractoriness between successive stimuli, we also clocked the prior stimulus in Experiment 3. RTs to all stimuli were required.

Five subjects were run. Two, rather than one, mobile spots now crossed the screen, the first (clocking the prior stimulus) passing the fixation point after $8 \mathrm{sec}$ and the second, $3 \mathrm{sec}$ later. An imperative toneburst $(88 \mathrm{~dB}, 1,000 \mathrm{~Hz}, 50 \mathrm{msec}$ duration) could occur on either of these occasions with a probability of .5 . Three blocks of 64 trials were given, with the first block being for practice. Nearly complete EP recovery after an interval of $8 \mathrm{sec}$ is generally found to be the case in the auditory modality (Bess \& Ruhm, 1972, with tone pairs, find complete recovery after $6 \mathrm{sec}$ ), whereas at $3 \mathrm{sec}$ both recovery function and fast habituation data indicate substantial refractoriness.

The results of Experiment 3 were disappointing. N1-P2 amplitude to the stimulus at the end of the spot's transit showed an amplitude depression when the prior stimulus occurred, relative both to its amplitude when the prior stimulus was absent $[\mathrm{t}(4)=5.9$, $\mathrm{p}<.001]$ and to N1-P2 amplitude to the prior stimulus itself $[\mathrm{t}(4)=3.8, \mathrm{p}<.01]$. The magnitude of refractoriness was, however, noted to be less than that typically found for this ISI. In a direct replication (Experiment 4) of Experiment 3 (i.e., with prior stimulus again randomized and made irrelevant), refractory effects were still obtained. Five subjects were run in this experiment.

\section{EXPERIMENT 5}

Experiments 3 and 4 show auditory refractoriness, unlike visual refractoriness, to reflect more than mere effects of temporal uncertainty. Despite the objective equivalence of stimuli, a larger response is evoked when the stimulus is the first event encountered in a trial. How may we explain this? Perhaps this advantage of the "first" stimulus is expressing the general sense in which the first of anything is special. Should this be the case, then with such "firstness" removed (by, for instance, having subjects regard the stimuli of Experiments 3 and 4 as constituting a single, long sequence of events rather than a series of trials), controlling temporal uncertainty should again be found to be fully effective in abolishing refractoriness.

A sense of continuity of stimulation was established 
by having the oscilloscope "clock" stimuli by revolving continuously around a circular course. Each orbit of the spot took $3 \mathrm{sec}$. Bleeps could occur whenever the moving spot passed the fixation point. Either one or three such cycles elapsed between stimuli, thus generating the $3-\sec$ (refractory) and 9-sec (full recovery) ISIs. One or three small spots appearing above the fixation point after each bleep indicated either the 3- or the 9-sec interval.

Six subjects were run. RTs to the bleeps were required. One hundred stimuli were presented in a single, long "clocked" sequence, with the initial 20 being for practice and the dissipation of "firstness." A control condition was also run, with an "unclocked" sequence having identical temporal structure, but with temporal uncertainty given full play by removal of the clock. This main part of the experiment is reported in detail in Wastell (1979). A further supplementary control has since been run. This control retained all aspects of the clocked condition, including the visual stimulation of the clock, except that by having the clock cycle (arbitrarily) every $6.7 \mathrm{sec}$, no predictive temporal information was provided. A deficiency in the original control was that, without the stationary spots to distinguish the 3- and 9-sec intervals, event uncertainty becomes confounded with ISI: Whether or not a stimulus will occur after $3 \mathrm{sec}$ is quite random, but once more than $3 \mathrm{sec}$ pass, a stimulus $6 \mathrm{sec}$ later is known to be certain. By retaining the stationary spots, the supplementary control condition avoided this problem.

In the original "unclocked" control condition, typical EP refractoriness at the short ISI was found: N1-P2 amplitude was depressed by $23 \%[\mathrm{t}(5)=3.31, \mathrm{p}<.01]$. In the supplementary control, N1-P2 was also depressed by a similar figure at the $3-\mathrm{sec}$ relative to the 9-sec ISI $[26 \% ; \mathrm{t}(4)=2.96, \mathrm{p}<.05]$. With the elimination of temporal uncertainty in the "clocked" condition, this decrease, however, did not occur: Values of 20.8 and 20.0 microV were obtained for the 3-and 9-sec intervals, respectively. Clocking also reduced overall N1-P2 amplitude, shortened median RT (by $60 \mathrm{msec}$ ), and reduced the latencies of both $\mathrm{N} 1$ and P2. Both N1 and P2 latencies were also increased at the longer ISI in the "unclocked" condition.

\section{GENERAL DISCUSSION}

The results of the experiments presented here are consistent with the view that the amplitude of the late componentry of the human EP is strongly determined by active information processing activities on the part of the subject. Although a notion of refractoriness is suggested by the procedural characteristics of temporal recovery and fast habituation studies, we show here that response decrements in such work are consistently underlain by variations in the temporal information delivered by stimuli. This conclusion is indirectly supported by an array of results from fast habituation studies, as has been argued elsewhere (Wastell, 1979). Behavioral data are also not consistent with a state of refractoriness prevailing for several seconds after stimulation (Welford, 1952).

The fact that refractory EP amplitude decrements are frequently associated with a forward shift in latency (see Experiment 5 here) further supports an explanation for such refractory effects in terms of temporal uncertainty and the active processing of information: Passive neural fatigue would surely produce a lengthening of response. That the late components of the EP are sensitive to temporal uncertainty is also reinforced by the increased overall amplitude (and delayed latency) of N1-P2 in the "unclocked" control of Experiment 5.

In Wastell (1978), the term "stimulus significance" was adopted to denote the total import of a stimulus for the organism, including its information content and such less easily defined qualities as "firstness." With the roots of the present work being much embedded in the habituation literature, the concept of a neuronal model of the world (Sokolov, 1975) was also taken on board. Information delivery is defined in these terms as reflecting the inadequacies of the neuronal model, and in temporal recovery and habituation experiments, we are thus arguing the neuronal model to be deficient primarily in its temporal aspects.

In terms of the concepts of stimulus significance and neuronal models, the findings of this paper may be recapitulated as showing EP refractoriness to be due to variations in stimulus significance. With stimulus significance brought fully under experimental control, the sensitivity of the EP to recent prior stimulation (this effect generically encompassing the phenomena of both fast habituation and temporal recovery) is abolished. In the visual modality, stimulus significance is primarily determined by the deficiencies of the neuronal model in its temporal aspects. In the auditory modality, though, a factor of "firstness" can also play a role in paradigms in which stimulus order has meaning.

We grant that all the experiments presented in this paper are based on a paradigm involving paired stimuli, yet refractory-like effects are obtained across a diverse spread of experimental procedures. However, it is our argument that all manifestations of EP refractoriness can logically be reduced to implying an amplitude depression due to recent stimulation, and it is this essence with which our studies are concerned.

On a final digression regarding the clock paradigm, its general value in exerting experimental control over temporal uncertainty is pointed out. Independently, Gottsdanker (1970) has employed a similar methodology (known as transit-signal methodology) in pure RT research in order to eliminate time uncertainty. To close this paper, we shall see how the degree of control afforded by the clock is reflected in concurrent slowwave activity. Data are presented (Figure 2) from one subject (run as a pilot for further work) using a standard S1-S2 contingent negative variation (CNV) paradigm (with S2 occurring with probability $.5, \mathrm{ISI}=2 \mathrm{sec}$ ) implemented both with and without "clocking." Inspection of Figure 2 shows that, following the usual orienting wave to $\mathrm{S} 1, \mathrm{CNV}$ with a linear clock (as in Experiments 2, 3, and 4) rises to its maximum only just before the time of delivery of $S 2$, whereas without clocking a state of maximum preparation is developed and maintained for a period of fully $1 \mathrm{sec}$ prior to S2. For both clocked and unclocked trials, when $\mathrm{S} 2$ is delivered, CNV resolves rapidly, contributing a positive wave in the ac record (Figure 2b) at the latency of P2. On the non-S2 clocked trials, for which the time of nonappearance of S2 is more or less precisely known via the clock, we see CNV slowly but surely falling away after the point of S2's nonocurrence. The relative slowness of this decay was found, by inspecting individual CNVs, not to be an artifact of averaging inhomogeneous single responses, but rather, to be a faithful reflection of a consistent morphology among the singletons. In the concurrent ac record (Figure $2 \mathrm{~b}$ ), this slowly resolving CNV 

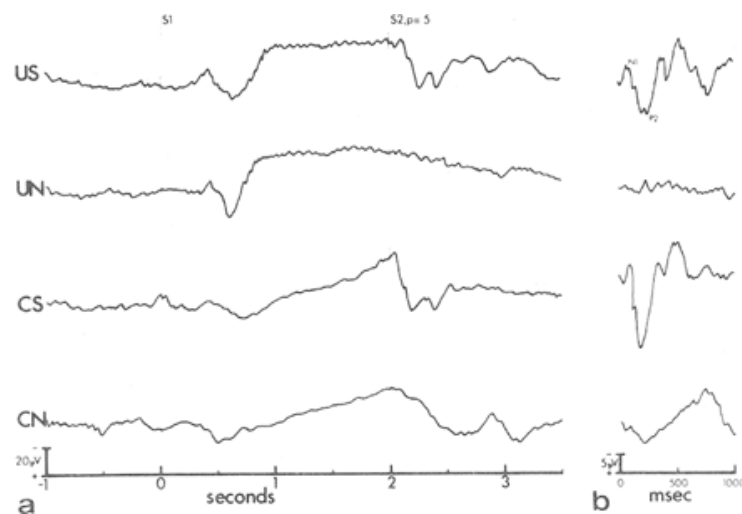

Figure 2. Part A shows dc brain activity (vertex electrode) associated with four conditions in the S1-S2 paradigm. US and $\mathrm{UN}=$ clocked, S2 present/absent, respectively. Similarly, CS and $\mathrm{CN}=$ clocked, $\mathrm{S} 2$ present/absent. Part $\mathrm{B}$ shows post-S2 ac records obtained by high-pass digital filtering.

shift is shown to give rise to a positive potential, which must correspond to the so-called "emitted" potential of the P300 literature. The relatively shallow amplitude of this phenomenon has been ascribed to its greater latency variability (Ruchkin \& Sutton, 1978). Research into this phenomenon should be aided using the clock paradigm to control such variability. The results presented here tentatively suggest (1) that resolving CNVs may contribute positivity to the ac record as early as P2, (2) that the emitted potential is an intrusion into the ac record of a resolving $\mathrm{CNV}$, and (3) that its shallower amplitude reflects a slower gradient of CNV resolution associated with stimulus omission.

\section{REFERENCES}

Bess, J. C., \& Ruнm, H. B. Recovery cycle of the acoustically evoked potential. Journal of Speech and Hearing Research, 1972, 15, 507-517.
Davis, H., Mast, T., Yoshie, N., \& Zerlin, S. The slow response of the human cortex to auditory stimuli: Recovery process. Electroencephalography and Clinical Neurophysiology, $1966,21,105-113$.

Fruhstorfer, H., Soveri, P., \& Jarvilehto, T. Short-term habituation of the auditory evoked response in man. Electroencephalography and Clinical Neurophysiology, 1970, 28, 153161.

Gottsdanker, R. A. A transit-signal methodology for studying reaction time. Behavior Research Methods \& Instrumentation, 1970, 2, 6-8.

McCarthy, G., \& Donchin, E. The effects of temporal and event uncertainty in determining the waveforms of the auditory event related potential (ERP). Psychophysiology, 1976, 13, 581590.

Ruchkin, D. S., \& Sutron, S. Emitted P300 potentials and temporal uncertainty. Electroencephalography and Clinical Neurophysiology, 1978, 45, 268-277.

Sokolov, E. N. The neuronal mechanisms of the orienting reflex. In E. N. Sokolov \& D. S. Vinogradava (Eds.), Neuronal mechanisms of the orienting reflex. Hillsdale, N.J: Erlbaum, 1975.

Wastell, D. G. Attention and the habituation of human brain potentials. Unpublished doctoral thesis, Durham University, 1978.

W ASTELL, D. G. Temporal uncertainty and the recovery function of the auditory EP. In Proceedings of the International Evoked Potentials Symposium, Nottingham (England). Lancaster: MTP Press, 1979.

Wastell, D. G., \& Kleinman, D. A psychoanatomical investigation of the locus of the mechanism responsible for the refractoriness of the visual vertex potential. Perception \& Psychophysics, 1980, 27, 149-152.

WELFORD, A. T. The "psychological refractory period" and the timing of high-speed performance-A review and a theory. British Journal of Psychology, 1952, 43, 2-19.

(Received for publication December 1, 1981.) 